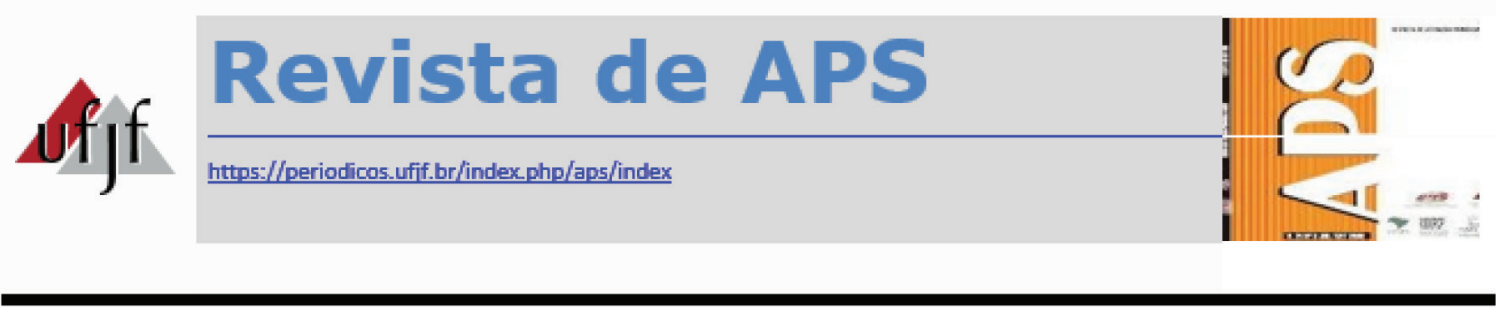

\title{
Acolhimento aos pacientes com necessidades de saúde mental na perspectiva dos profissionais da Atenção Primária à Saúde de Iguatu-CE
}

\section{Reception for patients with mental health needs from the perspective of primary health care professionals of Iguatu - CE}

\author{
Jomábia Cristina Gonçalves dos Santos ${ }^{1}$, Andressa Aires Alencar ${ }^{2}$, Tayrine Huana \\ de Sousa Nascimento ${ }^{3}$, Antonia Renata Lopes Lima ${ }^{4}$, Farley Janusio Rebouças \\ Valentim ${ }^{5}$
}

\begin{abstract}
RESUMO
A Atenção Primária à Saúde é a porta de entrada do Sistema Único de Saúde do Brasil, tendo o acolhimento como uma diretriz da Política de Humanização do SUS. Considerando que desde o processo de Reforma Psiquiátrica, os pacientes com necessidades em saúde mental podem ser acompanhados em seus territórios e através da atenção básica, este trabalho objetivou investigar como acontece o acolhimento e acompanhamento dos pacientes de saúde mental pelas equipes de atenção básica no município de Iguatu, Ceará. Para se chegar a esta compreensão, foram realizadas sete entrevistas semidiretivas com profissionais que atuam em equipes da Estratégia Saúde da Família neste município. Como método de análise de dados se utilizou a Análise de Conteúdo de Bardin. Os resultados foram agrupados e discutidos através de categorias e apontaram que os profissionais conhecem o que a literatura propõe acerca do acolhimento, entretanto existem dificuldades em inseri-lo nas práticas do cotidiano e trabalho.
\end{abstract}

PALAVRAS-CHAVE: Acolhimento. Saúde Mental. Atenção Primária à Saúde.

\footnotetext{
${ }^{1}$ Psicóloga; Especialista em Saúde da Família e da Comunidade pela Escola de Saúde Pública do Ceará; Especialista em Saúde Mental pela Faculdade Dom Alberto. E-mail: jomabia13@hotmail.com

${ }^{2}$ Cirurgiã-dentista; Especialista em Saúde da Família e Comunidade pela Escola de Saúde Pública do Ceará.

${ }^{3}$ Enfermeira; Especialista em Saúde Mental Coletiva pela Escola de Saúde Pública do Ceará; Especialista em Saúde da Família e da Comunidade pela Faculdade Dom Alberto.

${ }^{4}$ Psicóloga; Pós-graduanda em Intervenção ABA para o Autismo e Deficiência Intelectual pelo Child Behavior Institute of Miami- CBI; Servidora Pública do município de Sobral.

${ }^{5}$ Psicólogo; Mestre em Psicologia pela Universidade Federal do Ceará; Docente do Centro Universitário Christus UniChristus.
} 


\begin{abstract}
Primary Health Care is the gateway to the Brazilian Unified Health System (SUS). Reception is a guideline of the SUS Humanization Policy. Considering that since the Psychiatric Reform process, patients with mental health needs can be followed up in their territories and through primary care, this study aimed to investigate how the reception and monitoring of mental health patients happens by primary care teams in the municipality from Iguatu, Ceará. To reach this understanding, seven semi-directive interviews were carried out with professionals who work in teams of the Family Health Strategy in this municipality. As a method of data analysis, Bardin's Content Analysis was used. The results were grouped and discussed through categories. The results showed that the professionals know what the literature proposes about welcoming, however there are difficulties in inserting it in daily work practices.
\end{abstract}

KEYWORDS: Reception. Mental Health. Primary Health Care.

\title{
INTRODUÇÃO
}

A Atenção Primária à Saúde (APS) ou Atenção Básica se apresenta como porta de entrada do Sistema Único de Saúde (SUS) no Brasil e é considerada como o primeiro nível do processo contínuo de atenção à saúde, sendo responsável por coordenar os fluxos de atendimento dentro do serviço, acompanhamento longitudinal dos indivíduos, bem como garantir o acesso ao usuário conforme sua demanda, seguindo os princípios e diretrizes do SUS ${ }^{1}$.

Atualmente a APS é organizada através da Estratégia Saúde da Família (ESF), um modelo de atendimento comunitário que possui como foco a unidade familiar. Pela ESF é realizado um conjunto de ações de saúde individuais, familiares e coletivas que envolvem promoção, prevenção, proteção, diagnóstico, recuperação, vigilância em saúde, entre outras, devendo ser efetivadas por uma equipe multiprofissional lotada em um território específico e com uma população adstrita².

Durante os processos de fortalecimento da APS, em 2003, foi lançada a Política de Humanização do SUS, que propõe atuação seguindo posturas éticas, clínicas e políticas em suas formas de conduzir o trabalho em saúde. Uma das diretrizes dessa política é o acolhimento, que deve sustentar as relações entre equipe/serviço e usuários, devendo ser construído de forma coletiva objetivando estabelecer relações de confiança, compromisso e fortalecimento de vínculos ${ }^{3}$.

O processo de Reforma Psiquiátrica, que se iniciou em meados da década de 1970, vem buscando a desinstitucionalização no cuidado em saúde mental, propondo uma redução do número de leitos nos manicômios e a implementação de uma rede substitutiva comunitária de cuidados, na qual o paciente possa ser acompanhado em seu território. No atual modelo de saúde vigente no Brasil, espera-se que a APS seja também corresponsável pelos cuidados dispensados aos usuários com necessidades em 
saúde mental ${ }^{4}$.

Ribeiro, Caccia-bava e Lorenzi ${ }^{5}$ apontam que dentro da APS, as equipes de saúde da família se deparam cotidianamente com pacientes com necessidades de saúde mental, já que estes também estão inseridos em seus territórios, tendo como principais demandas os transtornos mais leves, como ansiedade e quadros depressivos.

Em Iguatu, nos territórios de abrangência cobertos pela equipe de apoio da estratégia saúde da família (NASF) Residente, do Programa de Residência Integrada em Saúde da Escola de Saúde Pública do Ceará (RIS/ESP-CE), os profissionais recebem constantemente demandas de saúde mental. Dito isso, este trabalho se propôs a investigar como acontece o acolhimento e acompanhamento dos pacientes com necessidades de saúde mental pelas equipes de atenção básica, buscando ainda compreender as concepções dos profissionais sobre acolhimento e as possíveis dificuldades encontradas na realização deste, bem como propor estratégias que objetivem amenizá-las.

\section{MATERIAL E MÉTODO}

Este trabalho constituiu-se em uma pesquisa de abordagem qualitativa, de natureza aplicada e cunho exploratório. Godoy ${ }^{6}$ afirma que a pesquisa de abordagem qualitativa parte de questões ou foco de interesses amplos, procurando entender o fenômeno segundo a perspectiva dos sujeitos.

De acordo com Marconi e Lakatos 7 , a pesquisa de caráter exploratório possui o objetivo de formulação de questões e problemas, familiarizando o pesquisador com o fenômeno, bem como o clarificando, o que se compõe característica dessa pesquisa. É ainda de natureza aplicada, pois tem como motivação a produção de conhecimento para aplicação de seus resultados, partindo de um embasamento teórico já existente ${ }^{8}$.

Caracterizou-se também como uma pesquisa de campo, que segundo Marconi e Lakatos? (p. 186) é "aquela utilizada com o objetivo de conseguir informações e/ou conhecimentos acerca de um problema para o qual se procura uma resposta, ou de uma hipótese que se queira comprovar", podendo ainda descobrir novos fenômenos durante a execução desta.

O público-alvo foram profissionais de duas equipes da Estratégia Saúde da Família do município de Iguatu-CE. Salienta-se que a escolha por essas equipes se deu devido ao fato de ser o campo de atuação da equipe de residentes da Turma V da RIS/ESP-CE, na qual a pesquisadora está lotada.

O município de Iguatu está localizado no centro-sul do estado do Ceará, a aproximadamente $380 \mathrm{~km}$ da capital de Fortaleza, com território estimado em 1.029,214 km² , densidade demográfica de 93,76 hab./ $/ \mathrm{km}^{2}$ e com a população estimada de 101.386 habitantes ${ }^{9}$. 
Atualmente o município conta com 31 equipes de Estratégia Saúde da Família, três Centros de Atenção Psicossocial (CAPS), outros dispositivos da Atenção Especializada e de Urgência e Emergência em Saúde, e ainda instituições que compõem o Sistema Único de Assistência Social (SUAS), a saber, Centro de Referência de Assistência Social (CRAS) e Centro de Referência Especializado de Assistência Social (CREAS).

A pesquisa possuiu amostragem não probabilística por conveniência, aquela que se configura pela escolha de participantes que estão mais acessíveis ao pesquisador e não são selecionados por um critério estatístico. No caso deste estudo, os participantes foram contatados e questionados sobre o interesse de participação no local de trabalho, sendo-Ihes também explicados os objetivos da pesquisa.

O trabalho contou com a participação de sete profissionais de saúde com diferentes níveis de escolaridade visto que a Política Nacional de Atenção Básica (PNAB) compreende o acolhimento como um conceito ampliado e transversal a todos os trabalhadores da atenção básica.

A amostra teve como critérios de inclusão: profissionais que estivessem durante o período da coleta de dados lotados nas referidas unidades de saúde e que possuíssem no mínimo quatro meses de experiência na APS, visto a relevância do tempo de prática no serviço para responder aos questionamentos. Os critérios de exclusão dizem respeito àqueles que não aceitaram participar da pesquisa, bem como os que estivessem em período de férias no momento da coleta.

A produção de dados foi realizada entre os meses de agosto e setembro de 2019, sendo utilizada como instrumento a entrevista semidiretiva. Para Manzini ${ }^{10}$, a entrevista semidiretiva tem o foco em um assunto sobre o qual se cria um roteiro com perguntas principais, que são complementadas por outras questões que podem surgir no momento da entrevista.

A entrevista se organizava da seguinte maneira: um bloco referente a perguntas sobre o acolhimento de um modo geral ( $O$ que você entende por acolhimento? Como é realizado o acolhimento na unidade de saúde em que você trabalha?), outro bloco sobre o acolhimento aos pacientes com necessidades de saúde mental (Como é realizado o acolhimento aos pacientes com necessidades de saúde mental na unidade de saúde em que você trabalha? Como se dá o acompanhamento desse usuário pela Estratégia Saúde da Família? Quais são os encaminhamentos realizados e/ou ofertados para esses pacientes durante o acompanhamento?) e, por fim, questões sobre dificuldades percebidas e estratégias de enfrentamento (Você percebe a existência de dificuldades em realizar o acolhimento e acompanhamento desses usuários na atenção básica? Se sim, quais são? Quais estratégias poderiam são utilizadas para auxiliar o profissional a realizar este acolhimento? Quais outras intervenções poderiam ser realizadas?).

Ainda como método de coleta de dados, a fim de controlar a quantidade de participantes, visto se tratar de uma pesquisa qualitativa, apresenta-se a Técnica de 
Saturação. É considerada saturada uma coleta de dados quando nenhum novo elemento é encontrado e o acréscimo de novas informações deixa de ser necessário, pois não altera a compreensão do fenômeno estudado ${ }^{11}$. Essa técnica é entendida como um critério que permite estabelecer a validade de um conjunto de dados.

As entrevistas foram realizadas nas próprias unidades de saúde e não tiveram tempo de duração estipulado. Foram, ainda, gravadas e posteriormente transcritas no momento da análise de dados. Após a transcrição, as gravações foram excluídas.

A análise dos dados foi realizada entre os meses de agosto de 2019 e janeiro de 2020, e o método utilizado foi a Análise de Conteúdo de Bardin. De acordo com Bardin ${ }^{12}$, a análise de conteúdo consiste em um conjunto de técnicas de análise das comunicações que se propõe a utilizar procedimentos sistemáticos de descrição do conteúdo coletado.

O projeto de pesquisa foi submetido ao comitê de Ética em Pesquisa - CEP - da Escola de Saúde Pública do Ceará, protocolo n CAAE: 15457019.5.0000.5037, obtendo parecer favorável, seguindo as normas da Resolução 466/12, que regulamenta a pesquisa com seres humanos. Ressalta-se ainda que a identidade dos participantes foi resguardada, tendo sido utilizada a letra "e" de entrevistado, seguida de número cardinal para indicar a fala dos participantes durante a apresentação e discussão dos resultados.

\section{RESULTADOS}

Foram entrevistados sete profissionais de saúde, sendo seis mulheres e um homem. Como aponta a literatura, as mulheres compõem o maior número de profissionais de saúde no Brasil ${ }^{13}$. No que diz respeito à idade, os participantes possuíam entre 27 e 65 anos, todos com mais de um ano de experiência na Atenção Primária a Saúde, tendo, alguns deles, mais de 15 anos de atuação no SUS.

As equipes das ESF eram compostas pelas seguintes categorias profissionais: enfermagem, medicina, odontologia, recepcionistas, técnicos de enfermagem, agentes comunitários de saúde (ACS), agentes de endemias, auxiliares de serviços gerais, que compunham as equipes mínimas, e ainda as profissionais do Núcleo Ampliado de Saúde da Família (NASF): psicóloga, assistente social, nutricionista e fisioterapeuta.

Dentre os participantes entrevistados, existiu representação das categorias: auxiliar de serviços gerais, enfermagem, odontologia, ACS e serviço social. Houve impasses para se realizar entrevistas com profissionais da categoria médica, devido a desencontros na agenda dos profissionais lotados nas unidades de saúde com a pesquisadora responsável, o que pode ser considerada uma fragilidade dessa pesquisa.

Os dados analisados através da Análise de Conteúdo de Bardin ${ }^{12}$ resultaram em três categorias, intituladas: “As concepções sobre acolhimento e sua prática no serviço de 
saúde", "Acolhimento e cuidado em saúde mental na APS", "Dificuldades e possibilidades de enfrentamento: pela integralidade do cuidado", que serão discorridas a seguir.

\section{DISCUSSÃO}

\section{As concepções sobre acolhimento e sua prática no serviço de saúde}

$\mathrm{Na}$ primeira categoria os entrevistados narraram suas compreensões sobre acolhimento e como eles o visualizam no seu cotidiano de trabalho. Ao serem indagados sobre em que consiste o acolhimento, os entrevistados responderam:

"Acolhimento é a recepção humanizada do paciente, saber recebê-lo, é saber repassar informações corretas né, saber de uma forma integrada todo o funcionamento da unidade básica de saúde pra poder ser repassado pra ele" (e. 3)

"O acolhimento é a atenção que você dá ao paciente quando ele chega na unidade, que ele, não necessariamente por uma profissional, mas multiprofissional, que você vai fazer uma escuta do paciente e daí você encaminha para os determinados serviços" (e. 4)

"O acolhimento é a parte que a profissional de saúde chega a praticar com o seu paciente né [...] acolhimento é você tratar bem, perguntar como está, é levar as localidades certas do atendimento, mostrar onde fica as salas corretas" (e. 5)

Em 2003, o Ministério da Saúde lançou a Política de Humanização do SUS - PNH, o HumanizaSUS, como uma nova proposta de estruturação da Atenção Primária. A PNH visa a uma maior comunicação entre gestores, trabalhadores e usuários como forma de enfrentamento das relações de poder que podem causar práticas desumanizadoras, bem como autonomia e a corresponsabilidade dos profissionais de saúde em seu trabalho e dos usuários no cuidado de $\mathrm{si}^{3}$.

Uma das diretrizes do HumanizaSUS é o acolhimento. De acordo com BRASIL ${ }^{3}$, "acolher é reconhecer o que o outro traz como legítima e singular necessidade de saúde", afirmando que o acolhimento deve sustentar as relações entre equipe/serviço e usuários, devendo ser construído de forma coletiva, objetivando relações de confiança, compromisso e fortalecimento de vínculos.

Ao dissertar sobre como realizar o acolhimento durante os processos de trabalho, a PNH ressalta que a escuta qualificada deve ser oferecida pelos trabalhadores aos usuários do serviço a fim de garantir o acesso às tecnologias adequadas às suas necessidades, ampliando e efetivando, assim, as práticas de saúde 3 .

O acolhimento surgiu como fundamental na reorganização da assistência nos serviços de saúde, modificando o modelo de atenção tecnoassistencial. O Ministério da Saúde afirma que o acolhimento deve ir além da recepção do usuário e envolver todos 
os profissionais do serviço, considerando as necessidades de saúde desde a entrada no sistema, entendendo-o como um recurso de apoio e qualificação, possibilitando ao usuário um cuidado justo e integral, reconhecendo que este acesso é um direito fundamental ${ }^{14}$.

No que diz respeito à compreensão do Acolhimento como uma das diretrizes da $\mathrm{PNH}$ e quais seriam seus objetivos, as respostas dos entrevistados condizem com o que aponta a literatura, entretanto ao dissertarem como essa diretriz é posta em prática, surgem em suas falas as contradições:

“[...] o acolhimento já não é lá essas coisas que inclusive é um dos grandes problemas de algumas unidades de saúde [...] tem muito paciente que não quer frequentar a UBS, deixa de usar o serviço por conta do acolhimento, ou porque teve uma experiência ruim naquele momento" (e. 1)

“Rapaz, de 0 a 10, eu acho que aqui tá em 4. Eu não acho que o acolhimento aqui dessa unidade seja bem feito" (e. 3)

"A gente foi capacitado, todos os profissionais da unidade são capacitados em acolhimento, mas na realidade ele não acontece de fato" (e. 4)

"Temos profissionais que é mais paciente, mais comprometido com o que fazem, que atendem de maneira é positiva, os pacientes, e temos profissionais um pouco mais difíceis no trato profissional" (e. 7)

Lopes et al. ${ }^{15}$ ressaltam a relevância da natureza das relações de reciprocidade entre usuários que têm necessidades de saúde e trabalhadores que reconhecem essas necessidades, havendo compreensão e significação de suas singularidades e de que a oferta dos saberes possibilita a existência de intervenções continuadas de vínculo, produção de autonomia e responsabilização pelo resultado.

Pelas falas dos entrevistados, existem impasses nas relações entre profissionais e usuários, existem formas de atendimento "positivas" e outras "mais difíceis". Lopes et al. ${ }^{15}$ dizem que quando acontecem falhas nesse contato inicial entre profissional e usuário, os processos e cuidado em saúde podem ficar comprometidos.

Ao relatarem o que acreditam serem os motivos da falha no acolhimento e dificuldades relacionais com os usuários em seus ambientes de trabalho, os entrevistados disseram:

"Eu acho que a falha no acolhimento aqui tá sendo pela falta da integralização do PSF [Programa Saúde da Família] que eu acho que não é muito integrada, acaba cada um ficando no seu quadrado, não sabendo repassar as informações devidas" (e. 3)

"O que a gente percebe é que o paciente chega, fica disperso, passar por um, por outro. Então ainda não aconteceu de fato, sabe. $O$ paciente ainda fica muito largado" (e. 4)

Bezerra e Carvalho ${ }^{16}$ apontam que problemas estruturais do serviço podem dificultar as relações de troca entre os profissionais de saúde e os 
usuários. Os autores relatam que, quando há falhas nas qualidades estruturais da unidade - demandas excessivas, vínculos frágeis de trabalho, falta de acesso e informações, entre outras -, ficam em risco a confiança e o respeito, e que isso afeta o cuidado ofertado.

Mitre, Andrade e Cotta $^{17}$ salientam que os profissionais tendem a praticar ações ritualizadas para com os usuários e que podem acabar resultando em conflitos relacionais. Os autores destacam que quanto menos ritualizadas as ações e mais flexíveis os profissionais, maior será a possibilidade de troca de saberes para realizar o acolhimento. Ressalta-se aqui que a PNH se propõe como forma de enfrentamento das relações de poder verticalizadas existentes entre profissionais e usuários.

É válido destacar a importância das reuniões de equipe, capacitações, repasse de informações e gestão compartilhada do cuidado para todos os profissionais que atuam nas unidades de saúde, visto que o efetivo acolhimento vai para além da oferta da prática curativa em si, mas também se faz presente no saber responder às dúvidas dos usuários e lhes dar os melhores direcionamentos dentro do serviço.

\section{Acolhimento e cuidado em saúde mental na APS}

Nessa categoria foram elencadas questões relativas ao acolhimento e cuidado ofertado aos pacientes com necessidades de saúde mental que fazem parte dos territórios de abrangência dos profissionais entrevistados. Sobre como é dispensado o acolhimento a esses usuários, os participantes disseram:

"Olha, é um pouco complicado porque a gente percebe que as pessoas têm muito preconceito com pacientes que tem algum transtorno mental, alguma necessidade de saúde mental, eles veem aquele paciente como usuário do CAPS e muitas vezes o médico renova a receita, não olha pro paciente [...] Eu não acho que eles são bem acompanhados. Eu acho que é feita uma coisa muito pra inglês ver" (e. 1)

"Só sei que aqui eles pegam a receita, mas outra coisa assim, eu não sei dizer não" (e. 2)

"E quando tem algum paciente de saúde mental [...] os que ainda têm como serem atendidos, os mais calmos, a gente ainda consegue atender tranquilamente, se não, a gente faz só uma referência pro CEO [Centro de Especialidades Odontológicas]" (e .3)

Desde a década de 1970, o processo de Reforma Psiquiátrica, que ocorreu em conjunto com a Reforma Sanitária, vem postulando conceitos e práticas na atenção aos transtornos mentais no Brasil, tendo como foco principal a desinstitucionalização, a redução do número de leitos nos manicômios e a implementação de uma rede substitutiva comunitária de cuidados ${ }^{4}$. 
Para constituir essa rede comunitária de cuidados, foram implantados, na década de 80, os Centros de Atenção Psicossocial (CAPS), tornando-se parte da rede de atenção secundária. Os CAPS são centros especializados no tratamento e acompanhamento de usuários com transtornos mentais graves e persistentes ${ }^{4}$.

A Rede de Atenção Psicossocial (RAPS) do município de Iguatu-CE é composta por CAPS III, que atende o público adulto, CAPS Ad, responsável pelo acompanhamento de usuários com uso excessivo e álcool e outras drogas, e CAPS infanto-juvenil, que atende pacientes menores de 18 anos de idade. Além desses equipamentos, a RAPS do município conta ainda com os demais equipamentos de saúde, dentre eles a APS e Residência Terapêutica. A Rede Intersetorial de Assistência e Educação e demais instituições comunitárias podem ser ativadas a fim de assegurar o cuidado em saúde mental.

Ao se pensar no acompanhamento de usuários com necessidades menos complexas ou aqueles que estão com o quadro e saúde mental mais estável, foram desenvolvidas, a partir de 2001, propostas de articulação entre saúde mental e atenção primária a saúde, a fim de uma assistência integral aos usuários ${ }^{18}$.

Compreendendo que o processo de Reforma Psiquiátrica propõe uma ruptura com o modelo hospitalocêntrico e predominância do saber psiquiátrico no atendimento dos sujeitos com necessidades de saúde mental, sabe-se da importância do acompanhamento dos indivíduos para além dos CAPS, já que se faz necessária a inserção destes em seus territórios, tornando-os parte da rede de cuidados dispensada ${ }^{19}$.

A Atenção Primária à Saúde tem como foco os seus territórios de abrangência, proporcionando atendimento integral a sua população adstrita ${ }^{20}$. Ao se tornar parte dessa rede de cuidados, espera-se que a APS acolha e ordene os cuidados aos usuários com necessidades de saúde mental através de toda a rede de atenção à saúde. Mas o que foi relatado durante as entrevistas é que esse cuidado em saúde mental na unidade básica de saúde ainda não acontece de forma efetiva:

"Na verdade, essa descentralização ainda não aconteceu de fato. Pra mim resumese aquele atendimento voltado, aquela atenção voltada pro medicamento, curativa" (e. 4)

"Eu acho que se dá de uma maneira legal. Passa aqui primeiro, o médico encaminha pros CAPS" (e. 5)

"Eu vejo que aqui no PSF [Programa Saúde da Família] é só mais receita. Minha receita, minha consulta, minha receita, minha consulta" (e. 7)

Um dos primeiros apontamentos feitos pelos entrevistados diz respeito ao preconceito para com os pacientes em saúde mental, que ainda são vistos como pessoas agressivas.

Em um recente estudo, Cassiano, Marcolan e Silva ${ }^{21}$ apontam que o atendimento a indivíduos com algum transtorno mental na APS é realizado, em algumas situações, com 
atitude de resistência e distanciamento, sendo essa conduta fruto de uma inadequada formação prévia em saúde mental e de estigma e preconceito por parte de profissionais de saúde. Tais condutas podem gerar nos usuários sentimentos de exclusão e um possível afastamento da instituição de saúde.

Ainda nessa pesquisa, os autores pontuam que a escuta qualificada, compreensão, empatia entre paciente com demanda de saúde mental e profissional de saúde geram junto ao usuário autonomia e corresponsabilização do cuidado, o que é proposto pela Reforma Psiquiátrica. Entretanto, as ações de saúde mental na APS vêm se constituindo apenas em triagem, renovação de receita e encaminhamento para o serviço especializado ${ }^{21}$.

Com relação à prática de renovação de receitas, Campos, Bezerra e Jorge ${ }^{22}$ ressaltam que essa conduta na APS favorece a utilização indiscriminada de medicações sem a compreensão sobre a verdadeira necessidade de utilização pelo usuário. Entende-se, hoje, que o acompanhamento em saúde mental está para além de uso de medicamentos e que existe uma particularidade e subjetividade dos sujeitos que se implicam no processo de adoecimento mental, que necessitam de escuta qualificada de caráter terapêutico para se alcançar integralidade desse cuidado ${ }^{23}$.

Minóia e Minozzo ${ }^{24}$, ao dissertarem sobre o acolhimento a pacientes de saúde mental na atenção básica, identificaram que os profissionais, geralmente, têm dificuldade em conduzir o acolhimento dos usuários, não sabendo como manejar a escuta e compreender a importância de o usuário refletir sobre o que está falando, entre outras questões, e acabam por voltar-se para uma conduta prescritiva e de encaminhamento para atenção secundária, os CAPS.

Bezerra et al. ${ }^{25}$ apontam que a "renovação de receitas" é resultado do processo de medicalização que se faz presente na atualidade, o qual está intimamente relacionado com a prática de assistência biomédica e modelo curativo que a Reforma Psiquiátrica vem tentando transformar. O poder biomédico coloca a medicação como única alternativa para o enfrentamento do adoecimento psíquico, patologizando comportamentos e retirando a autonomia e corresponsabilização do usuário no tratamento, visto que o medicamento passa a ser o centro do cuidado.

A saúde mental na APS precisa envolver mais do que apenas o cuidado aos transtornos mentais, sendo importante a construção de modelos de cuidado integrais que abordem o usuário, sua família e a comunidade em geral, por isso a importância da inserção dos usuários em atividades em seus territórios ${ }^{22}$. Sobre isso, os entrevistados contam:

"Eu sugiro que participe do nosso grupo de idosos, vejo questão de pele, tudinho. Por que o que a gente observa é que o paciente de saúde mental, a gente só olha pra ele como o paciente de saúde mental” (e. 4) 
"O médico encaminha pros CAPS, né, pros CRAS e de lá ele continua o tratamento" (e. 5)

"Então, às vezes o paciente vem com um encaminhamento de saúde mental e você percebe dermatoses, outras questões, desnutrição, que precisariam de uma atenção interdisciplinar, e não necessariamente só a saúde mental e sim de um olhar de uma forma integral pra esse paciente" (e. 7)

Apesar dos impasses, os participantes também dissertaram sobre os encaminhamentos e a necessidade do olhar integral aos usuários de saúde mental atendidos nas unidades de saúde. Faz-se importante encaminhar, quando necessário, sem se desresponsabilizar pelo cuidado².

É relevante inserir esses usuários em grupos, pois ações como estas proporcionam formação de vínculos reais e contínuos entre profissionais e usuários, ampliando as possibilidades de desenvolvimento de autonomia, autoestima, autocuidado ${ }^{22}$.

No território em que as ESF dos entrevistados estão localizadas existe uma rede comunitária de cuidados em que esses pacientes podem ser encaminhados, a saber: Academia de Saúde do bairro, onde ocorrem grupos de atividade física facilitados por um profissional, grupo do Centro de Referência de Assistência Social (CRAS), espaços idealizados pelos próprios usuários, como instituições religiosas, associações comunitárias, e ainda um grupo de caminhada intitulado "Prosa e Saúde", que é facilitado por profissionais residentes em saúde, mas que surgiu a partir uma iniciativa da população.

\section{Dificuldades e possibilidades de enfrentamento: pela integralidade do cuidado}

A terceira categoria objetiva apontar o que os profissionais dissertaram como dificuldades que podem ser as causas desses impasses, bem como quais estratégias podem ser efetivadas a fim de promover um melhor atendimento aos pacientes.

Ao serem questionados se percebiam a existência de dificuldades na realização do acolhimento e acompanhamento desses usuários pela APS, os entrevistados responderam:

"Eu já tive bastante receio. Receio de não tá tendo a conduta certa, tipo, é, será que eu sei qual é o encaminhamento real que ele tá precisando no momento" (e. 1)

"Existe. Existe muitas dificuldades. Despreparo da parte das pessoas que trabalham aqui" (e. 5)

"Principalmente a questão do despreparo dos profissionais, como eu já disse, eles têm medo, tem insegurança, é, pouco conhecimento sobre as questões de saúde mental" (e. 7)

Os discursos apontam que os profissionais não se sentem capacitados para acolher com segurança os usuários que os procuram com demandas de saúde mental, 
afirmando que durante seus processos de graduação obtiveram conhecimento superficial sobre o tema.

Campos Júnior e Amarante $^{26}$ identificaram em seu estudo que a maioria dos profissionais não se sentem seguros para lidar com as situações de saúde mental, justificando isso devido à falta de contato teórico, o que produz retrocessos nas formas de cuidado dispensadas e impossibilitam intervenções.

Ainda sobre essa questão, Jorge, Souza e $\mathrm{Franco}^{27}$ afirmam que a formação dos profissionais, o pouco conhecimento e a capacitação insuficiente para atuarem junto aos casos de transtornos mentais estão relacionados com a inexistência de práticas de saúde mental na Atenção Primária. Quinderé et al. ${ }^{28}$ também postulam que essa falta de capacitação e instrumentalização dos profissionais para intervir na saúde mental acabam gerando encaminhamentos desnecessários para os CAPSs, uma sobrecarga desnecessária e elevação nesses serviços.

Quando indagados no que os profissionais acreditavam que poderia auxiliar a efetivação do cuidado dispensado a esses pacientes, os mesmos ressaltaram:

"Eu acho que antes de tudo deve ser feitas capacitações [...] no sentido de orientar quanto a conteúdo mesmo" (e. 1)

"Acho que a estratégia básica mesmo seria a educação permanente. Educação permanente com todos os profissionais" (e. 3)

"[...] que a gente tenha o matriciamento na unidade" (e. 4)

"Eu sugiro assim, eu acho que sempre ter reuniões né, capacitações, e motivação também" (e. 5)

Assim, para que as ações da Saúde Mental sejam desenvolvidas na APS, é fundamental a capacitação dessas equipes por meio de atividades permanentes de discussão de casos com equipes de saúde mental, permitindo estratégias de cuidado que considerem as múltiplas determinações do processo saúdedoença e apoiem a superação do modelo manicomial no cuidado às pessoas com transtornos mentais ${ }^{22}$.

Como ferramenta de produção de conhecimento no trabalho, temos a Política Nacional de Educação Permanente do SUS - PNEPS, instituída em 2004, que representa um marco para a formação e trabalho no Brasil. A PNEPS coloca que os trabalhadores da saúde necessitam de constante atualização para concretização do cuidado e assistência aos usuários. A Educação Permanente em Saúde se propõe a traçar reflexões acerca do processo de trabalho nas instituições e saúde, visto que é baseada na aprendizagem significativa e na possibilidade de transformar as práticas de trabalho instituídas nos serviços de saúde ${ }^{29}$.

Assim, a Educação Permanente em Saúde consiste na produção e sistematização de conhecimentos relativos à formação e ao desenvolvimento para a atuação em saúde, 
envolvendo práticas de ensino, diretrizes didáticas e orientação curricular, bem como reflexões baseadas na experiência em serviço ${ }^{29}$.

Se em Iguatu, existe um nó com relação ao acolhimento e cuidado aos pacientes em saúde mental, e se os profissionais apontam a necessidade de capacitações e estudo sobre o tema, não seria uma proposta a se pensar, em nível de gestão? Seja gestão das unidades em que estes profissionais estão lotados ou até mesmo gestão municipal.

Sabe-se que a falha no acolhimento impacta significativamente a qualidade do atendimento prestado ao usuário, pois impossibilita o acesso a um cuidado justo, ampliado e integral. As reflexões sobre como enfrentar essas dificuldades precisam ser feitas por todos os envolvidos, a fim de uma melhoria nos processos de trabalho e visando ao estabelecimento de uma relação de confiança entre usuário, profissional, equipe e gestão ${ }^{14}$.

Outro ponto em destaque na fala dos entrevistados diz respeito ao apoio matricial para fortalecimento da integralidade do cuidado:

“[...] trazer o CAPS pra cá, pra visitar junto com a gente" (e. 5)

"[...] aquela reuniãozinha que tinha, o matriciamento, eu a gente tinha, até isso sumiu, que era tão bom" (e. 6)

"E não só a equipe dos médicos, enfermeiros, né vocês profissionais, mas também pra gente, tanto atendente, como agente de saúde. Uma equipe só" (e. 7)

O Ministério da Saúde anseia que haja integração entre a equipe do Programa de Saúde da Família e os profissionais da Rede de Apoio de Saúde Mental (RAPS), e reestrutura a assistência psiquiátrica, indicando a existência de equipes matriciais de apoio à saúde da família, para uma maior efetivação das práticas em saúde mental. 0 Apoio Matricial é um arranjo organizacional que visa conceder suporte técnico em áreas específicas às equipes responsáveis pelo desenvolvimento de ações básicas de saúde para a população ${ }^{30}$.

O apoio matricial em saúde mental pode ser dispensado tanto pelos profissionais dos CAPS, quanto daqueles que compõem o Núcleo Ampliado de Saúde da Família (NASF). Costumam ser trabalhados no matriciamento conteúdos relativos a acolhimento, encaminhamento, intervenção medicamentosa, reinserção e reabilitação psicossocial, assistência domiciliar e familiar e ações territoriais ${ }^{31}$.

Como um tipo de arranjo organizacional, com o objetivo de responder ao princípio da integralidade, a prática do matriciamento almeja ampliar e qualificar o escopo de ações das equipes da APS. Desse modo se propõe a integrar saúde mental e saúde geral, para que esses usuários não sejam assistidos somente na rede de atenção especializada ${ }^{31}$. 
Como foi dito pelos entrevistados, o matriciamento nas unidades de saúde em que trabalham não está acontecendo, talvez devido à superlotação dos CAPS, ou ao número de profissionais que compõem essas equipes, falta de transporte, entre outros motivos.

Os profissionais apontam em suas falas algumas possibilidades de enfrentamento, contudo é importante que a gestão municipal seja contatada, pois algumas ações necessitariam que os gestores compreendessem a importância de se fortalecer o acolhimento em todas as redes de saúde. Será que os gestores possuem esse entendimento?

Estratégias de Educação Permanente, recursos para transporte e aumento da quantidade de profissionais são questões que precisam do envolvimento de todos os profissionais, usuários e gestão, pois o acolhimento não se esgota na Atenção Primária à Saúde e deve perpassar por todos os equipamentos do município.

\section{CONCLUSÃO}

As tecnologias de cuidado em saúde mental necessitam de constante renovação e reflexão, a fim de se alcançar aquilo que é proposto pela Reforma Psiquiátrica: cuidado integral aos usuários em seus territórios com promoção, autonomia, reabilitação psicossocial e corresponsabilização no tratamento. Para isso se faz necessária a transformação do modelo biomédico e curativista que ainda está presente nos serviços de saúde.

O acolhimento, enquanto diretriz da Política de Humanização do SUS, é apontado como uma das possibilidades de enfrentamento desse modelo verticalizado de cuidado. Acolher diz respeito a escutar o que o usuário sabe sobre seu processo de adoecimento, compreendendo que é a partir do diálogo que, juntos, profissional e paciente podem traçar o melhor planejamento terapêutico, e que ambos são responsáveis pelo tratamento em saúde.

Através desta pesquisa, pôde-se constatar que, apesar de conhecerem o que a literatura aponta acerca do acolhimento, colocá-lo em prática nas instituições de saúde ainda é um desafio constante, e que práticas cristalizadas e instrumentalizadas estão presentes nas relações entre profissionais e usuários. No que diz respeito ao acolhimento em saúde mental, isso ainda se intensifica, pois os profissionais se sentem despreparados para atenderem essas demandas, construindo um cuidado fragmentado, por vezes limitado à renovação de receitas e encaminhamentos para os serviços especializados.

Os entrevistados relataram conhecimento dessas falhas nas práticas dispensadas e propuseram algumas estratégias de enfrentamento, estando no centro dessas ações a Educação Permanente em Saúde, em conjunto com o apoio matricial. 
A utilização dessas outras tecnologias em saúde pode possibilitar a construção de um cuidado longitudinal, multidimensional considerando a subjetividade e autonomia dos sujeitos.

Por fim, faz-se necessário o investimento na qualificação dos profissionais da Atenção Primária à Saúde. É válido destacar que os dados demonstrados através desta pesquisa serão debatidos com as equipes das unidades de saúde, usuários e gestão do município, a fim de que, talvez, algo do que foi aqui apresentado possa ser considerado para fortalecimento das práticas de assistência em saúde mental dispensadas pelos profissionais da atenção básica de Iguatu.

\section{REFERÊNCIAS}

1. Filho JAS, Bezerra AM. Acolhimento em Saúde Mental na atenção primária a saúde: Revisão Integrativa. Revista Multidisciplinar e de Psicologia. 2018; 12(40):613-27.

2. Ministério da Saúde (Brasil). Política Nacional de Atenção Básica. 2017 [acesso em 2020 abr 23] Disponível em: http://bvsms.saude.gov.br/bvs/saudelegis/gm/2017/ prt2436_22_09_2017.html.

3. Ministério da Saúde (Brasil). Política Nacional de Humanização. Brasília, Distrito Federal; 2003.

4. Amarante $P$, Nunes MO. A reforma psiquiátrica no SUS e a luta por uma sociedade sem manicômios. Revista Ciência e Saúde Coletiva. 2018; 23(6):2067-74.

5. Ribeiro MOP, Caccia-bava MCGG, Lorenzi CG. Atenção à saúde mental na estratégia saúde da família: recursos não reconhecidos. Revista Psicologia USP. 2013; 24(3):369-90.

6. Godoy AS. Introdução à pesquisa qualitativa e suas possibilidades. Revista de Administração de Empresas. 1995; 35(2):57-63.

7. Marconi MA, Lakatos EM. Fundamentos de Metodologia Científica. 5a ed. São Paulo: Editora Atlas; 2013.

8. Vilaça MLC. Pesquisa e ensino: considerações e reflexões. Revista escrita. 2010; $1(2): 59-74$.

9. Instituto Brasileiro de Geografia e Estatística [homepage na internet]. Censo demográfico 2015: Contagem da População; resultados preliminares da amostra. [acesso em 2020 ago 12]. Disponível em: http://cod.ibge.gov.br/1E4T.

10. Manzini EJ. A entrevista na pesquisa social. Didática. 1990; 26-27:149-158.

11. Nascimento LCN, Souza TV, Oliveira ICS, Moraes JRMM, Aguiar RCB, Silva LF. Saturação teórica em pesquisa qualitativa: relato de experiência na entrevista com escolares. Rev. Bras. Enferm. 2018; 71(1):228-33. 
12. BARDIN, L. Análise de conteúdo. 3ạ edição. Lisboa: Edições 70; 2006.

13. Poz MRD, Perantoni CR., Girardi S. Formação, mercado de trabalho e regulação da força de trabalho em saúde no Brasil. In FUNDAÇÃO OSWALDO CRUZ. A saúde no Brasil em 2030 - prospecção estratégica do sistema de saúde brasileiro: organização e gestão do sistema de saúde [online]. 2013; 3:187-233.

14. Coutinho LRP, Barbieri AR, Santos MLM. Acolhimento na atenção primária a saúde: uma revisão integrativa. Revista Saúde Debate. 2015; 39(105):514-24.

15. Lopes AS, Vilar RLA, Melo RHV, França RCS. O acolhimento na Atenção Básica em saúde: relações de reciprocidade entre trabalhadores e usuários. Revista Saúde Debate. 2015; 39(4):114-23.

16. Bezerra RS, Carvalho EL. Profissionais de saúde e dispositivos de mediação na atenção básica. In: Pinheiro R, Martins PH. Usuários, redes sociais, mediações e integralidade em saúde. Rio de Janeiro: CEPESC: IMS-UERJ; Recife: Editora Universitária UFPE; São Paulo: ABRASCO; 2011. p.149-60.

17. Mitre SM, Andrade EIG, Cotta RMM. Avanços e desafios do acolhimento na operacionalização e qualificação do Sistema Único de Saúde na Atenção Primária: um resgate da produção bibliográfica do Brasil. Revista Ciência\&Saúde Coletiva. 2012; 17(8):2071-85.

18. Tanaka OU, Ribeiro EL. Ações de saúde mental na atenção básica: caminho para a ampliação da integralidade da atenção. Revista Ciência e Saúde Coletiva. 2009; 14(2):477-86.

19. Ministério da Saúde (Brasil). Reforma Psiquiátrica e política de saúde mental no Brasil. Brasília, 2005.

20. Andrade LOM, Barreto ICHC, Bezerra RC. Atenção Primária a Saúde e Estratégia Saúde da Familia. In Campos GWS, et al. Tratado de Saúde Coletiva. 1a reimpressão (1a edição: 2006). São Paulo: Editora FioCruz; 2009.

21. Cassiano APC, Marcolan JF, Silva DA. Atenção primária à saúde: estigma a indivíduos com transtornos mentais. Rev enferm UFPE on line. 2019 [acesso em 2020 abr 23]. Disponível em: https://periodicos.ufpe.br/revistas/revistaenfermagem/article/ view/239668/32517.

22. Campos DB, Bezerra IC, Jorge MSB. Tecnologias do cuidado em saúde mental: práticas e processo na Atenção Primária. Revista Brasileira de Enfermagem. 2018; 71(5):2228-36

23. Alencar TOS, Cavalcante EAB, Alencar BR. Assistência Farmacêutica e saúde mental no Sistema Único de Saúde. Rev Ciênc Farm Básica. 2012; 33(4):2025-37.

24. Minóia NP, Minozzo F. Acolhimento em Saúde Mental: Operando Mudanças na Atenção Primária à Saúde. Revista Psicol. Ciênc. Prof. 2015; 35(4):1340-49. 
25. Bezerra IC, Jorge MSB, Gondim APS, Lima LL, Vasconcelos MGF. "Fui lá no posto e o doutor me mandou foi pra cá": processo de medicamentalização e (des)caminhos para o cuidado em saúde mental na Atenção Primária. Revista Interface. 2014; 18(48):61-74.

26. Campos Jr A, Amarante PDC. Estudo sobre práticas de cuidado em saúde mental na Atenção Primária: o caso de um município do interior do estado do Rio de Janeiro. Cad. saúde colet. [online]. 2015; 23(4):425-35.

27. Jorge MSB, Sousa FSP, Franco TB. Apoio matricial: dispositivo para resolução de casos clínicos de saúde mental na Atenção Primária à Saúde. Rev Bras Enferm [Internet]. 2013; 66(5):738-44.

28. Quinderé PHD, Jorge MSB, Nogueira MSL, Costa LFA, Vasconcelos MGF. Acessibilidade e resolubilidade da assistência em saúde mental: a experiência do apoio matricial. Revista Ciênc Saúde Colet [Internet]. 2013; 18(7):2857-61.

29. BRASIL. Ministério da Saúde. Secretaria de Gestão do Trabalho e da Educação na Saúde. Departamento de Gestão da Educação na Saúde. Política Nacional de Educação Permanente em Saúde: o que se tem produzido para o seu fortalecimento? / Ministério da Saúde, Secretaria de Gestão do Trabalho e da Educação na Saúde, Departamento de Gestão da Educação na Saúde. 1ạ ed. rev. Brasília: Ministério da Saúde; 2018.

30. Correira VR, Barros S, Colvero LA. Saúde mental na atenção básica: prática da equipe de saúde da família. Rev. esc. enferm. USP [online]. 2011; 45(6):1501-06.

31. Lima M, Dimenstein M. O apoio matricial em saúde mental: uma ferramenta apoiadora da atenção à crise. Revista Interface. 2016; 20(58):625-35.

Submissão: abril de 2020.

Aprovação: agosto de 2020. 